\title{
Bénard convection with rotation and a periodic temperature distribution
}

\author{
S. J. D. D’Alessio \& K. A. Ogden \\ Department of Applied Mathematics, University of Waterloo, Canada
}

\begin{abstract}
This study investigates the effects of a sinusoidally varying temperature distribution and rotation on free convection between two rigid plates. The domain is assumed to be much larger in the horizontal direction than in the vertical direction, which naturally introduces a small parameter. An approximate analytical solution for the steady-state flow is derived by expanding the flow variables in the small parameter. The steady-state solution was also determined numerically using the commercial CFD software package CFX. A comparison of the results shows that the form of the steady-state flow pattern is indeed captured by the approximate analytical solution. Unsteady numerical calculations are also carried out for various sets of parameters to determine when the flow destabilizes, how the modulated temperature boundary condition and rotation affect the critical Rayleigh number, and also to illustrate the flow pattern that develops when the flow becomes unstable.
\end{abstract}

Keywords: Bénard convection, rotation, sinusoidally varying boundary condition, shallow flow, analytical, numerical.

\section{Introduction}

The problem of free convection within a long, rotating rectangular domain driven by a sinusoidally varying bottom temperature is studied. This problem is an extension of traditional Bénard convection to account for both rotation and a periodic temperature distribution along the bottom plate. Numerous studies have been devoted to Bénard convection. One previous related study is the work by Pascal and D'Alessio [1], which addresses the stability of the flow with rotation and a quadratic equation of state. Schmitz and Zimmerman [2] studied the effects of a spatially varying temperature condition together with wavy boundaries, but 
without rotation. They also simplified their governing equations by assuming a very large Prandtl number. The current work, on the other hand, takes advantage of the thinness of the fluid layer to simplify the governing equations. Other related work includes that of Malashetty and Swamy [3], who consider the effects of rotation and a temperature boundary condition which varies with time, and that of Basak et al. [4], who study the flow resulting from a spatially varying temperature boundary condition in a square cavity without rotation.

One goal of this research is to compare the steady and unsteady flows to that of traditional Bénard convection (henceforth referred to as the basic case) so as to assess the combined impact of rotation and a periodic temperature condition on the stability of the flow. Another objective is to contrast the approximate analytical steady-state solution with a fully numerical solution obtained from a commercial CFD software package.

\section{Governing equations}

The two-dimensional flow is confined to a thin rectangular domain shown in figure 1 . The domain is allowed to rotate about the $z$-axis and the flow is assumed to be uniform in the $x$-direction.

The governing equations are given by

$$
\begin{gathered}
\vec{\nabla} \cdot \vec{v}=0 \\
\frac{\partial \vec{v}}{\partial t}+(\vec{v} \cdot \vec{\nabla}) \vec{v}+f \hat{k} \times \vec{v}=\frac{-1}{\rho_{0}} \vec{\nabla} p-\frac{\rho}{\rho_{0}} g \hat{k}+\nu \nabla^{2} \vec{v} \\
\frac{\partial T}{\partial t}+(\vec{v} \cdot \vec{\nabla}) T=\kappa \nabla^{2} T
\end{gathered}
$$

where $\vec{v}=(u, v, w)$ is the velocity, $T$ is the temperature, $\vec{\Omega}=(f / 2) \hat{k}$ is the rate of rotation, $\vec{g}=-g \hat{k}$ is the acceleration due to gravity, and the density, $\rho$, is taken

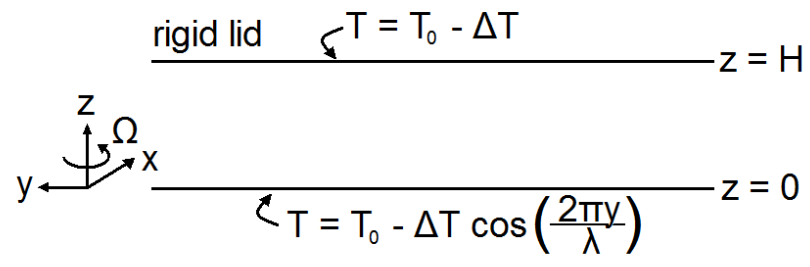

Figure 1: The coordinate system and flow configuration. 
to vary with temperature according to

$$
\rho=\rho_{0}\left(1-\alpha\left[T-T_{0}\right]\right),
$$

with $\alpha$ denoting the thermal expansion coefficient and $\rho_{0}, T_{0}$ representing reference values for density and temperature. The remaining fluid properties $\nu, \kappa$ refer to the kinematic viscosity and thermal diffusivity, respectively.

Since the flow is assumed to be uniform in the $x$-direction, the continuity equation permits a stream function, $\psi$, to be defined such that

$$
v=\frac{\partial \psi}{\partial z} \text { and } w=-\frac{\partial \psi}{\partial y} .
$$

The system of equations (1)-(5) can be expressed in terms of the stream function and the $x$-component of vorticity, $\zeta$, as follows

$$
\begin{gathered}
\zeta=-\left(\frac{\partial^{2} \psi}{\partial y^{2}}+\frac{\partial^{2} \psi}{\partial z^{2}}\right) \\
\frac{\partial \zeta}{\partial t}+\frac{\partial}{\partial y}\left(-\frac{\partial \psi}{\partial z} \frac{\partial^{2} \psi}{\partial y^{2}}+\frac{\partial \psi}{\partial y} \frac{\partial^{2} \psi}{\partial y \partial z}\right)-\frac{\partial}{\partial z}\left(\frac{\partial \psi}{\partial z} \frac{\partial^{2} \psi}{\partial y \partial z}-\frac{\partial \psi}{\partial y} \frac{\partial^{2} \psi}{\partial z^{2}}\right) \\
-f \frac{\partial u}{\partial z}=\alpha g \frac{\partial T}{\partial y}+\nu\left(\frac{\partial^{2} \zeta}{\partial y^{2}}+\frac{\partial^{2} \zeta}{\partial z^{2}}\right), \\
\frac{\partial u}{\partial t}+\frac{\partial \psi}{\partial z} \frac{\partial u}{\partial y}-\frac{\partial \psi}{\partial y} \frac{\partial u}{\partial z}-f \frac{\partial \psi}{\partial z}=\nu\left(\frac{\partial^{2} u}{\partial y^{2}}+\frac{\partial^{2} u}{\partial z^{2}}\right) \\
\frac{\partial T}{\partial t}+\frac{\partial \psi}{\partial z} \frac{\partial T}{\partial y}-\frac{\partial \psi}{\partial y} \frac{\partial T}{\partial z}=\kappa\left(\frac{\partial^{2} T}{\partial y^{2}}+\frac{\partial^{2} T}{\partial z^{2}}\right) .
\end{gathered}
$$

These equations are to be solved in the rectangular region spanning one wavelength in the $y$-direction subject to the no-slip and prescribed temperature conditions given by

$$
\begin{gathered}
u=v=w=0 \text { at } z=0, H \text { and } y=0, \lambda, \\
T=T_{0}-\Delta T \cos \left(2 \pi \frac{y}{\lambda}\right) \text { at } z=0, T=T_{0}-\Delta T \text { at } z=H, y=0, \lambda .
\end{gathered}
$$

The corresponding boundary conditions for $\psi$ become

$$
\psi=\frac{\partial \psi}{\partial z}=0 \text { at } z=0, H \text { and } \psi=\frac{\partial \psi}{\partial y}=0 \text { at } y=0, \lambda
$$

It is noted that the vorticity lacks conditions. This can be resolved by utilizing the over-specified conditions for $\psi$ and applying Green's Second Identity

$$
\int_{V}\left(\phi \nabla^{2} \chi-\chi \nabla^{2} \phi\right) d V=\int_{S}\left(\phi \frac{\partial \chi}{\partial n}-\chi \frac{\partial \phi}{\partial n}\right) d S,
$$

where $\phi$ and $\chi$ denote arbitrary differentiable functions, $\frac{\partial}{\partial n}$ is the normal derivative, and $S$ is the surface enclosing the volume $V$. Choosing $\phi$ to satisfy 
$\nabla^{2} \phi=0$ and letting $\chi \equiv \psi$, then $\nabla^{2} \chi=\nabla^{2} \psi=-\zeta$. Also, applying the boundary conditions $\frac{\partial \psi}{\partial n}=\psi=0$ on $S$, the above leads to integral constraints for the vorticity given by

$$
\int_{0}^{H} \int_{0}^{\lambda} \phi_{n} \zeta d y d z=0
$$

where

$$
\phi_{n}(y, z)=e^{ \pm 2 n \pi z}\left\{\begin{array}{c}
\sin (2 n \pi y) \\
\cos (2 n \pi y)
\end{array}\right\} \text { for } n=1,2,3, \cdots
$$

\subsection{Non-dimensionalization}

The governing equations and associated conditions are non-dimensionalized using the following scaling

$$
\begin{gathered}
t \rightarrow \frac{H^{2}}{\kappa} t, y \rightarrow \lambda y, z \rightarrow H z, \psi \rightarrow \kappa \psi, \zeta \rightarrow \frac{\kappa}{H^{2}} \zeta \\
T \rightarrow\left(T_{0}-\Delta T\right)+\Delta T T, u \rightarrow \frac{\kappa}{H} u
\end{gathered}
$$

This introduces the non-dimensional parameters

$$
R a=\frac{\alpha g H^{3} \Delta T}{\nu \kappa}, R o=\frac{\kappa}{H f \lambda}, \operatorname{Pr}=\frac{\nu}{\kappa}, \delta=\frac{H}{\lambda} .
$$

Cast in dimensionless form, the equations now become

$$
\begin{gathered}
\frac{\partial \zeta}{\partial t}-\delta \frac{\partial}{\partial z}\left(\frac{\partial \psi}{\partial z} \frac{\partial^{2} \psi}{\partial y \partial z}-\frac{\partial \psi}{\partial y} \frac{\partial^{2} \psi}{\partial z^{2}}\right)+\delta^{3} \frac{\partial}{\partial y}\left(-\frac{\partial \psi}{\partial z} \frac{\partial^{2} \psi}{\partial y^{2}}+\frac{\partial \psi}{\partial y} \frac{\partial^{2} \psi}{\partial y \partial z}\right) \\
-\frac{\delta}{R o} \frac{\partial u}{\partial z}=\delta \operatorname{Pr} R \frac{\partial T}{\partial y}+\operatorname{Pr}\left(\delta^{2} \frac{\partial^{2} \zeta}{\partial y^{2}}+\frac{\partial^{2} \zeta}{\partial z^{2}}\right) \\
\zeta=-\left(\delta^{2} \frac{\partial^{2} \psi}{\partial y^{2}}+\frac{\partial^{2} \psi}{\partial z^{2}}\right) \\
\frac{\partial u}{\partial t}+\delta\left(\frac{\partial \psi}{\partial z} \frac{\partial u}{\partial y}-\frac{\partial \psi}{\partial y} \frac{\partial u}{\partial z}\right)-\frac{\delta}{R o} \frac{\partial \psi}{\partial z}=\operatorname{Pr}\left(\delta^{2} \frac{\partial^{2} u}{\partial y^{2}}+\frac{\partial^{2} u}{\partial z^{2}}\right) \\
\frac{\partial T}{\partial t}+\delta\left(\frac{\partial \psi}{\partial z} \frac{\partial T}{\partial y}-\frac{\partial \psi}{\partial y} \frac{\partial T}{\partial z}\right)=\delta^{2} \frac{\partial^{2} T}{\partial y^{2}}+\frac{\partial^{2} T}{\partial z^{2}}
\end{gathered}
$$

while the boundary conditions are

$$
\begin{gathered}
u=\psi=\frac{\partial \psi}{\partial z}=0 \text { at } z=0,1 \text { and } u=\psi=\frac{\partial \psi}{\partial y}=0 \text { at } y=0,1, \\
T=1-\cos (2 \pi y) \text { at } z=0 \text { and } T=0 \text { at } z=1, y=0,1,
\end{gathered}
$$


and the integral constraints become

$$
\int_{0}^{1} \int_{0}^{1} \phi_{n} \zeta d y d z=0
$$

where

$$
\phi_{n}(y, z)=e^{ \pm 2 n \pi \delta z}\left\{\begin{array}{c}
\sin (2 n \pi y) \\
\cos (2 n \pi y)
\end{array}\right\} \text { for } n=1,2,3, \cdots
$$

\section{Steady and unsteady solutions}

The steady-state solution corresponding to the problem considered in this investigation is more complicated than that pertaining to the basic case due to the presence of a horizontal temperature gradient. Two methods are used to determine the steady-state solution. The first is an approximate analytical solution found by expanding the flow variables in a series in the small parameter $\delta$, while the second is a numerical solution obtained using a commercial CFD software package.

\subsection{Approximate analytical solution}

For small $\delta$, an approximate analytical solution can be constructed by expanding the flow variables in the following series

$$
\begin{gathered}
\psi=\psi_{0}+\delta \psi_{1}+\cdots, \\
\zeta=\zeta_{0}+\delta \zeta_{1}+\cdots, \\
u=u_{0}+\delta u_{1}+\cdots, \\
T=T_{0}+\delta T_{1}+\cdots .
\end{gathered}
$$

With the understanding that the Rossby $(R o)$ and Prandtl $(P r)$ numbers are $O(1)$, and the Rayleigh $(R a)$ number is $O(1 / \delta)$, the leading-order unsteady problem becomes

$$
\begin{gathered}
\frac{\partial T_{0}}{\partial t}=\frac{\partial^{2} T_{0}}{\partial z^{2}} \\
\frac{\partial \zeta_{0}}{\partial t}=\operatorname{Pr} \frac{\partial^{2} \zeta_{0}}{\partial z^{2}}+\operatorname{Pr} \delta R a \frac{\partial T_{0}}{\partial y} \\
\frac{\partial^{2} \psi_{0}}{\partial z^{2}}=-\zeta_{0} \\
\frac{\partial u_{0}}{\partial t}=\operatorname{Pr} \frac{\partial^{2} u_{0}}{\partial z^{2}}
\end{gathered}
$$

subject to

$$
u_{0}=\psi_{0}=\frac{\partial \psi_{0}}{\partial z}=0 \text { at } z=0,1
$$




$$
T_{0}=1-\cos (2 \pi y) \text { at } z=0 \text { and } T_{0}=0 \text { at } z=1,
$$

the integral constraint

$$
\int_{0}^{1} \zeta_{0} d z=0
$$

and the initial conditions

$$
\zeta_{0}=\psi_{0}=u_{0}=0 .
$$

Taking the initial temperature to be the steady-state solution given by

$$
T_{0}=(1-z)(1-\cos (2 \pi y)),
$$

it follows that the temperature remains constant for all time. It is also easily deduced that $u_{0}=0$. Thus, rotation does not affect the solution at leading order.

A straight-forward calculation yields the solution

$$
\begin{aligned}
\zeta_{0}(y, z, t)= & -\pi \delta R a\left(z^{2}-\frac{z^{3}}{3}-\frac{7 z}{10}+\frac{1}{10}\right) \sin (2 \pi y) \\
& +\sum_{n=1}^{\infty} a_{n} e^{-n^{2} \pi^{2} P r t} \cos (n \pi z), \\
\psi_{0}(y, z, t)= & \pi \delta R a\left(\frac{z^{4}}{12}-\frac{z^{5}}{60}-\frac{7 z^{3}}{60}+\frac{z^{2}}{20}\right) \sin (2 \pi y) \\
& -\sum_{n=1}^{\infty} \frac{a_{n}}{n^{2} \pi^{2}} e^{-n^{2} \pi^{2} \operatorname{Prt}}(1-\cos (n \pi z)),
\end{aligned}
$$

where

$$
a_{n}=2 \pi \delta R a \sin (2 \pi y) \int_{0}^{1}\left(z^{2}-\frac{z^{3}}{3}-\frac{7 z}{10}+\frac{1}{10}\right) \cos (n \pi z) d z
$$

for $n=1,2,3, \cdots$. It is worth noting that it is impossible for $\psi_{0}$ to satisfy all the boundary conditions; although the condition $\psi_{0}=0$ at $z=1$ is not exactly satisfied, all the other ones are.

As $t \rightarrow \infty$, the following leading-order steady-state solution emerges

$$
\begin{aligned}
& \zeta_{s}=-\pi \delta R a\left(z^{2}-\frac{z^{3}}{3}-\frac{7 z}{10}+\frac{1}{10}\right) \sin (2 \pi y), \\
& \psi_{s}=\pi \delta R a\left(\frac{z^{4}}{12}-\frac{z^{5}}{60}-\frac{7 z^{3}}{60}+\frac{z^{2}}{20}\right) \sin (2 \pi y) .
\end{aligned}
$$

The steady-state solution reveals a non-zero background flow, which results from the horizontal temperature gradient; recall that for the basic case the background flow is stationary. Dimensionalized plots showing the leading-order 

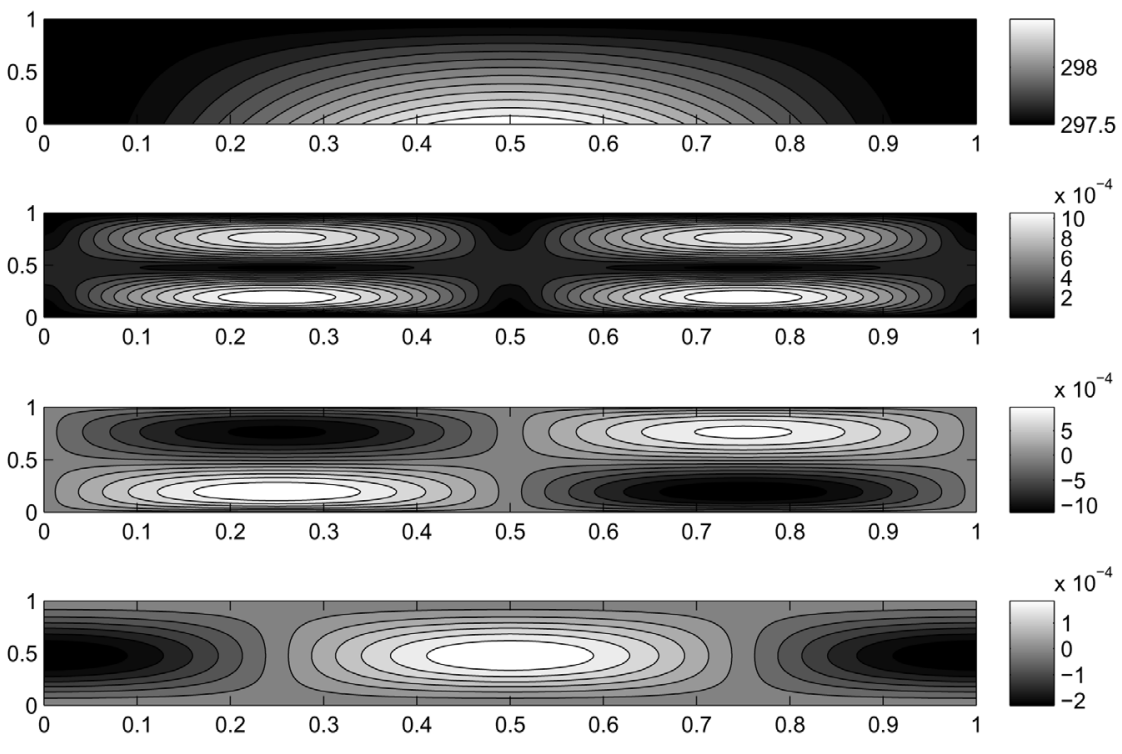

Figure 2: Leading-order solution for: $T_{0}$ (top) in $\mathrm{K}$, speed $\left(\left|\vec{v}_{0}\right|\right)$ (second from top), $v_{0}$ (second from bottom), and $w_{0}$ (bottom), in $\mathrm{m} / \mathrm{s}$.

steady-state solution are shown in figure 2, and indicate that two circulation cells develop. The simulations correspond to air at $298 \mathrm{~K}$ in a domain having a length of $20 \mathrm{~cm}$ and a height of $2 \mathrm{~cm}$. The temperature difference is $\Delta T=0.5 \mathrm{~K}$. The values of the non-dimensional parameters are $R a=388.7, \operatorname{Pr}=0.7046$, and $R o=0.0548$.

\subsection{Steady-state numerical simulations}

The steady-state solution is calculated numerically using the CFD software package CFX which solves the full Navier-Stokes and energy equations for arbitrary values of the non-dimensional parameters. In these simulations periodicity conditions are applied at the left and right boundaries of the domain. Results for two steady-state simulations will be discussed. The first corresponds to the basic case, while the second illustrates the effects of a sinusoidally varying bottom temperature and rotation. The parameter values are the same as those used in figure 2 for the analytical solution (i.e. $\operatorname{Ra}=388.7, \operatorname{Pr}=0.7046$ and $R o=0.0548$ ). Plotted in figure 3 are the temperature distributions for the two cases. It should be noted that the range of temperatures is smaller for the basic case because the bottom temperature for this case is taken to be the average value of the second case. Plots of the magnitude of the velocity $(|\vec{v}|)$ for each case are shown in figure 4 . It should be noted that while the velocity is not exactly zero for the basic case, it is two orders of magnitude smaller than that in the second case. Contour 


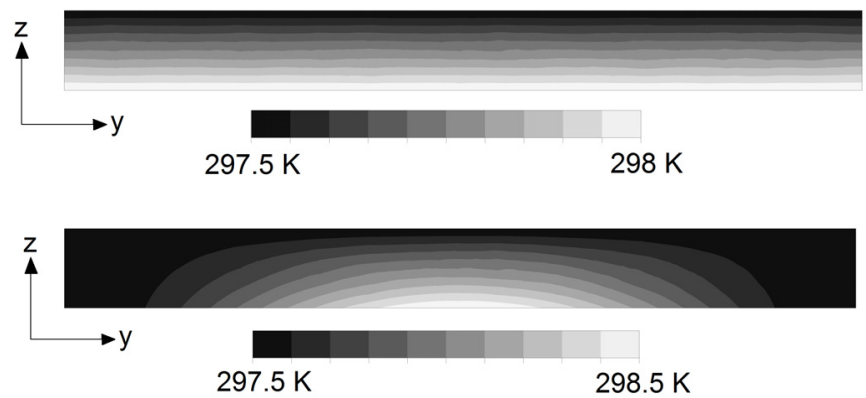

Figure 3: Steady-state temperature distribution for: basic case (top), and sinusoidal bottom temperature with rotation (bottom).
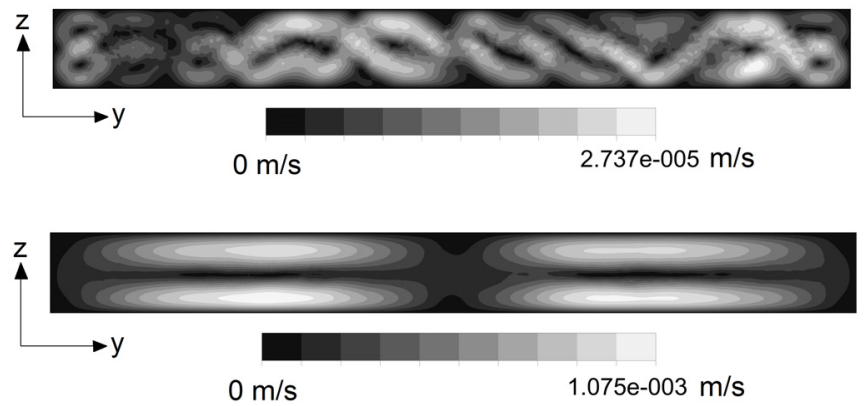

Figure 4: Steady-state plots of $|\vec{v}|$ for: basic case (top), and sinusoidal bottom temperature with rotation (bottom).

plots of the $v$ and $w$ velocity components for the case having a sinusoidally varying bottom temperature and rotation are displayed in figure 5. The $v$ and $w$ velocity components illustrate the circulation resulting from the horizontal temperature gradient. Comparing figure 2 with figures 3-5, it is clear that the leading-order analytical solution is in good agreement with the fully numerical solution, both in the qualitative flow pattern and also in the magnitude of temperature and velocities.

\section{Unsteady numerical simulations and flow stability}

The flow pattern that develops for unstable cases was also numerically investigated using CFX. As before, the basic case is also presented for comparison purposes. Shown in figure 6 is the basic case for an unstable case having $\Delta T=4 \mathrm{~K}$, $R a=3109$ and $\operatorname{Pr}=0.7046$.

Figure 6 clearly shows that 5 pairs of counter-rotating cells develop. Since the length of the domain is ten times its height, each cell forms a square. Figure 7 

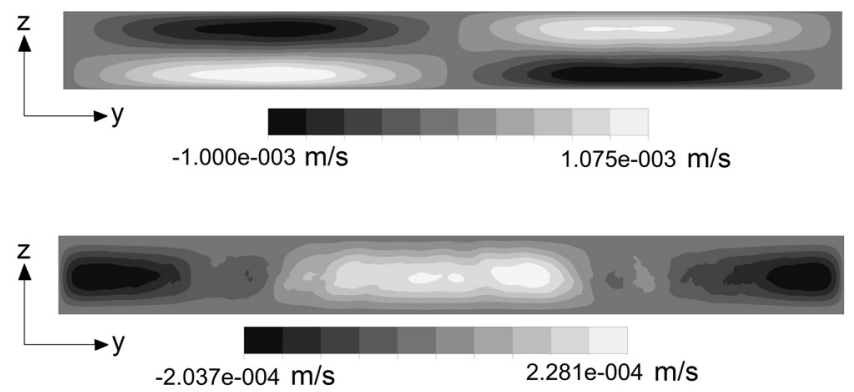

Figure 5: Steady-state velocity contour plots for the case with a sinusoidal bottom temperature and rotation: $v$ velocity component (top), $w$ velocity component (bottom).
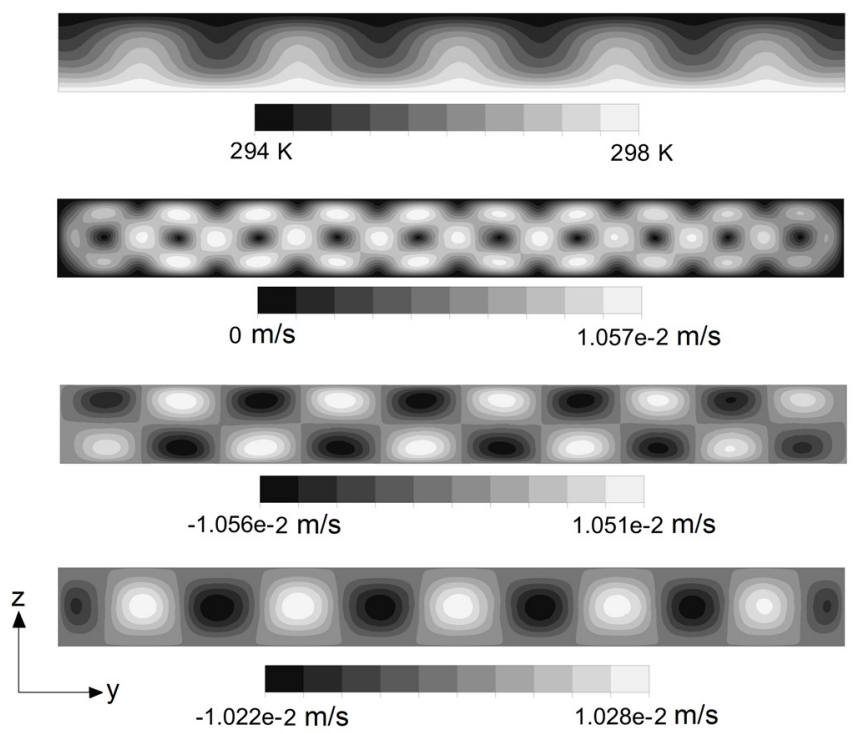

Figure 6: Unsteady case with a constant bottom temperature and no rotation: temperature (top), speed ( $|\vec{v}|)$ (second from top), $v$ (second from bottom), and $w$ (bottom).

shows the corresponding case with a sinusoidal bottom temperature and rotation $(R o=0.0548)$. The flow patterns are noticeably different from those shown in figures 3-5. Here, the flow destabilizes and breaks up into a greater number of cells; the number of cells was found to depend on $\Delta T$. Velocity plots are presented in figure 8 for both cases and for various temperature differences, and hence 


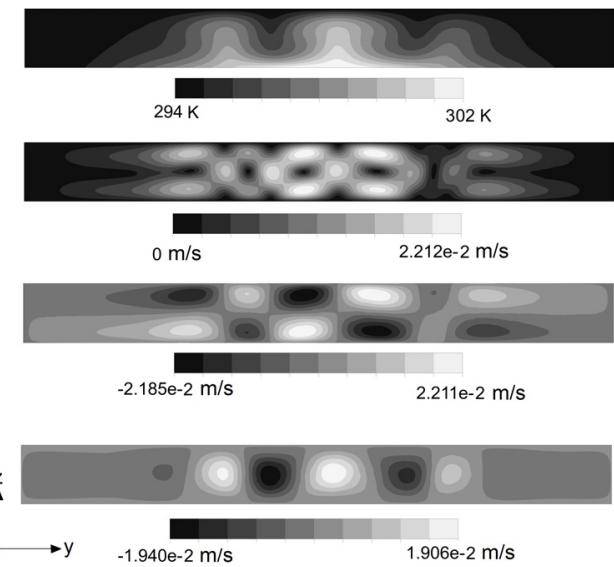

Figure 7: Unsteady case with a sinusoidally varying bottom temperature and rotation: temperature (top), speed $(|\vec{v}|)$ (second from top), $v$ (second from bottom), and $w$ (bottom).

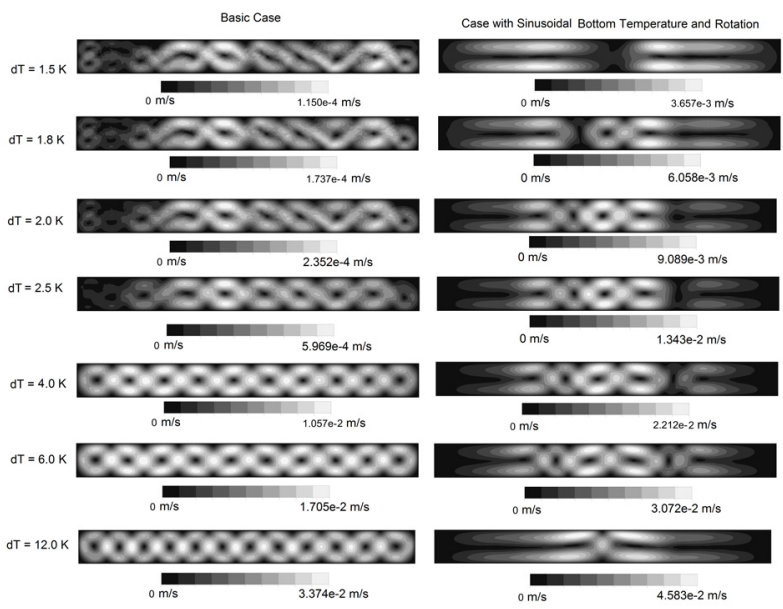

Figure 8: Speed $(|\vec{v}|)$ plots for the basic case and for bottom temperature variation with rotation, for increasing Rayleigh numbers $(\operatorname{Pr}=0.7046$ and $R o=0.0548$ for the case with rotation).

Rayleigh numbers. The results suggest that for the case with bottom temperature variation, the flow becomes unstable for $\Delta T$ between $1.5 \mathrm{~K}$ and $1.8 \mathrm{~K}$, while for the basic case this occurs for $\Delta T$ between $2.5 \mathrm{~K}$ and $4.0 \mathrm{~K}$. Since Pascal and D'Alessio [1] showed that rotation stabilizes Bénard convection, this indicates that the impact of bottom temperature variation significantly influences the stability 
of the flow. An interesting feature occurs when $\Delta T=12 \mathrm{~K}$ for the case with variable bottom temperature. The apparent trend is that increasing $\Delta T$ increases the number of cells that develop in an unstable flow; however, once the temperature difference is sufficiently large, the flow pattern resembles that of a stable flow with two cells, despite the fact that the circulation is intensified.

\section{Summary}

The combined effect of a sinusoidal bottom temperature and rotation on the background flow and on the stability of free convective flow was investigated. Contrary to Bénard convection, a non-zero background flow ensues. This background flow was determined analytically by taking advantage of the relative thinness of the fluid layer, and performing an expansion in terms of the thinness parameter. The background flow was also determined numerically using a commercial CFD software package. The leading-order analytical solution was found to be in good agreement with the numerical solution for stable cases. The impact on the stability of the flow was then investigated through numerical simulations. Although rotation has previously been found to stabilize the flow, variations in the bottom temperature was found to dominate and destabilize the flow when compared to Bénard convection. Lastly, interesting features emerging from unstable numerical simulations were also presented and discussed.

\section{References}

[1] Pascal, J.P. \& D'Alessio, S.J.D., The effects of density extremum and rotation on the onset of thermal instability, International Journal of Numerical Methods for Heat \& Fluid Flow 13, pp. 266-285, 2003.

[2] Schmitz, R \& Zimmerman, W., Spatially periodic modulated Rayleigh-Bénard convection, Physical Review E 53, pp. 5993-6011, 1996.

[3] Malashetty, M.S., \& Swamy, M., Effect of thermal modulation on the onset of convection in a rotating fluid layer, International Journal of Heat and Mass Transfer 51, pp. 2814-2823, 2008.

[4] Basak, T., Roy, S. \& Balakrishnan, A.R., Effects of thermal boundary conditions on natural convection flows within a square cavity, International Journal of Heat and Mass Transfer 49, pp. 4525-4535, 2006. 